\title{
Comparison of anesthetic effects of dexmedetomidine and tramadol, respectively, combined with propofol in percutaneous microwave coagulation therapy for hepatocellular carcinoma
}

\author{
CHUNQIN CHU $^{1 *}$, XUANLONG YI $^{1 *}$, JIAN SUN $^{1}, \mathrm{XI} \mathrm{ZHANG}^{1}, \mathrm{SULI} \mathrm{LIU}^{2}$, \\ NANNAN ZHANG ${ }^{1}$ and JUNTAO WANG ${ }^{1}$ \\ Departments of ${ }^{1}$ Anesthesiology and ${ }^{2}$ Ultrasound, The Affiliated Hospital of Qingdao University, \\ Qingdao, Shandong 266000, P.R. China
}

Received October 24, 2018; Accepted July 3, 2019

DOI: $10.3892 /$ ol.2019.10717

\begin{abstract}
Anesthetic effects and safety of dexmedetomidine and tramadol, respectively, combined with propofol in ultrasound-guided percutaneous microwave coagulation therapy (PMCT) for hepatocellular carcinoma (HCC) were compared. One hundred and seventy-six patients with HCC, treated by ultrasound-guided PMCT in The Affiliated Hospital of Qingdao University from January 2014 to December 2016, were retrospectively analyzed and divided into two groups: dexmedetomidine group (anesthetized with dexmedetomidine combined with propofol, $n=91$ ) and tramadol group (anesthetized with tramadol combined with propofol, $\mathrm{n}=85$ ). Changes in heart rate (HR), mean arterial pressure (MAP), and oxygen saturation $\left(\mathrm{SpO}_{2}\right)$ were recorded before oxygen inhalation (T1), intraoperationally (T2), and at 30 min postoperatively (T3), and the recovery time (recorded from the moment the use of anesthetic drugs stopped), hospital stay, visual analogue scale (VAS) score at $48 \mathrm{~h}$ after surgery, as well as the adverse reactions in the perioperative period were compared between the two groups. $\mathrm{HR}$ and $\mathrm{SpO}_{2}$ in the dexmedetomidine group at T2 and T3 were significantly lower than those in the tramadol group $(\mathrm{P}<0.05)$. HR and $\mathrm{SpO}_{2}$ at T2 were significantly lower than those at T1 and T3, and HR at T3 was lower than that at T1 $(\mathrm{P}<0.05)$. MAP in the dexmedetomidine group at $\mathrm{T} 2$ was significantly lower than that in the tramadol group $(\mathrm{t}=3.836, \mathrm{P}<0.001)$. MAP
\end{abstract}

Correspondence to: Dr Nannan Zhang or Dr Juntao Wang, Department of Anesthesiology, The Affiliated Hospital of Qingdao University, 16 Jiangsu Road, Shinan, Qingdao, Shandong 266000, P.R. China

E-mail: n978uq@163.com

E-mail: wangjuntao1228@163.com

*Contributed equally

Key words: dexmedetomidine, tramadol, propofol, microwave ablation, hepatocellular carcinoma at T2 was significantly lower than those at T1 and T3, and MAP at T3 was lower than that at T1 $(\mathrm{P}<0.05)$. The number of patients with shivering in the dexmedetomidine group was significantly higher than that in the tramadol group $(\mathrm{P}<0.05)$. Both tramadol and dexmedetomidine, respectively combined with propofol in PMCT for HCC surgery can achieve satisfactory anesthetic effects. However, tramadol combined with propofol is more effective in stabilizing the vital signs with less side-effects, and is more suitable for PMCT in patients with HCC than dexmedetomidine combined with propofol, which is worth popularizing and applying in clinic.

\section{Introduction}

Hepatocellular carcinoma (HCC) is a common malignancy, accounting for $7 \%$ of all cancers worldwide, with $>700,000$ deaths per year (1-3). Due to the lack of effective biomarkers in early primary HCC and the limited treatment options, it has become one of the most fatal cancers in the world and is a disease of great concern to the medical community $(4,5)$. Percutaneous radiofrequency ablation and percutaneous microwave coagulation therapy (PMCT) are the main techniques for treating patients with unresectable liver cancer or those who cannot tolerate surgery $(6,7)$. Surgical resection is widely accepted as an option for the treatment of HCC, however surgical morbidity and mortality have contributed to the development of minimally invasive ablation techniques over the past 20 years. Moreover, due to the improvement of technology and image guidance, PMCT has been a widely used thermal ablation method $(8,9)$. However, due to its high local temperature, patients suffer from a strong sense of discomfort (6), therefore, the appropriate and safe choice of anesthetic drugs is critical.

Dexmedetomidine is a new $\alpha_{2}$ adrenergic receptor agonist with high selectivity and special pharmacological effects that is widely used in clinical practice (10). Tramadol, as a commonly used drug for reducing chills in the clinic, can inhibit the hypothalamic thermoregulatory center and play a role in regulating the body temperature when binding to opioid receptors. Compared with other opioid sedatives, tramadol has a milder respiratory depression response $(11,12)$. At present, 
there are few reports on the application of tramadol in the treatment of HCC anesthesia with PMCT. Therefore, retrospective analysis of HCC patients treated with PMCT, and comprehensive comparison of the anesthetic effects of dexmedetomidine and tramadol, respectively, combined with propofol in PMCT for HCC treatment, were conducted in this study in order to provide a reference for clinical application of PMCT in the treatment of HCC.

\section{Patients and methods}

Data of patients. One hundred and seventy-six patients with HCC, treated by ultrasound-guided PMCT in The Affiliated Hospital of Qingdao University (Qingdao, China) from January 2014 to December 2016, were retrospectively analyzed and divided into two groups: 91 patients anesthetized with dexmedetomidine combined with propofol were assigned to the dexmedetomidine group, with an age range of 35-76 years and an average age of $56.72 \pm 4.28$ years; 85 patients anesthetized with tramadol combined with propofol were assigned to the tramadol group, with an age range of 31-77 years and an average age of $58.29 \pm 4.14$ years. Inclusion criteria: patients with complete clinical data; patients with indications for PMCT; patients receiving no relevant treatment in other hospitals. Exclusion criteria: patients with stage III-IV in TNM or tumor infiltrating; patients with allergic reactions to the drug used in this study; patients with contraindications to PMCT; patients during pregnancy and lactation; patients with acute gastrointestinal bleeding or other tumors; patients with no obvious abnormalities in kidneys and coagulation function; patients with disorders of communication or cognitive impairment. The study was approved by the Ethics Committee of The Affiliated Hospital of Qingdao University. The patients and their families signed the informed consent form and cooperated with the medical staff to complete the relevant diagnosis and treatment.

Methods. The dosage of anesthetic drugs was set according to patients' weight and age. Considering that the adverse reactions of each drug could affect the patients' heart rate (HR), mean arterial pressure (MAP) and oxygen saturation $\left(\mathrm{SpO}_{2}\right)$, the dosage of the anesthetic drugs in the study was strictly in accordance with the standard clinical operations (13). Patients were requested to abstain from consuming any food or drink for 8 or $4 \mathrm{~h}$, respectively, prior to the treatment. After entering the treatment room, the patients were connected to the monitor (Wuhan Kaijin Medical Technology Co., Ltd.) and they received nasal catheter oxygen inspiration $(3 \mathrm{l} / \mathrm{min})$ after opening veins pass. Patients in the dexmedetomidine group were intravenously injected with $0.5 \mu \mathrm{g} / \mathrm{kg}$ of dexmedetomidine (Sichuan Guorui Pharmaceutical Co., Ltd.; SFDA approval no. H20143195) by micro pump, and the patients in the tramadol group were intravenously injected with $100 \mathrm{mg}$ tramadol diluted with $10 \mathrm{ml}$ of $0.9 \%$ sodium chloride solution (Shanghai Xudong HaiPu Pharmaceutical Co., Ltd.; SFDA approval no. H20023785). The patients in both groups were treated with intravenous maintenance anesthesia until 3-5 min before the end of PMCT. After ultrasound-guided localization, propofol (1-2 $\mathrm{mg} / \mathrm{kg}$ ) was administered for intravenous induction, and the dose and speed of the pump were adjusted according to the patient's condition and maintained until the completion of PMCT.

Changes in $\mathrm{HR}, \mathrm{MAP}$, and $\mathrm{SpO}_{2}$ were detected before oxygen inhalation (T1), intraoperationaly (T2), and at $30 \mathrm{~min}$ postoperatively (T3). The recovery time (recorded from the moment the use of anesthetic drugs stopped), hospital stay, visual analogue scale (VAS) score at $48 \mathrm{~h}$ after surgery, and the adverse reactions during the perioperative period were compared between the two groups.

Statistical analysis. SPSS 19.1 software (IBM Corp.) was used for statistical analysis. The enumeration data were expressed as percentage [n (\%)], and the differences between two groups were compared by Chi-square test. The measurement data were expressed as mean \pm standard deviation, and the differences between two groups were compared by t-test. The data at multiple time points within each group were compared by repeated measures ANOVA with Least Significant Difference post hoc test. $\mathrm{P}<0.05$ was considered to indicate a statistically significant difference.

\section{Results}

Comparison of clinical data of patients. Comparative analysis was conducted in order to make the results accurate and reliable. The results showed that there was no significant difference in sex, age, weight, alcohol abuse, number of tumors, cirrhosis and TNM staging between the two groups ( $\mathrm{P}>0.05)$, suggesting that there was a comparability between the two groups of patients (Table I).

Analysis of HR in the dexmedetomidine and the tramadol group at different time points. The HR of patients in the dexmedetomidine group at T1, T2 and T3 was 83.52 \pm 8.39 , $72.45 \pm 8.12$ and $79.36 \pm 7.37$ beats/min, respectively. HR of patients in the tramadol group at different time points was $84.12 \pm 7.85,81.68 \pm 8.64$ and $82.95 \pm 7.91$ beats $/ \mathrm{min}$, respectively. There was no significant difference in HR between the two groups at $\mathrm{T} 1(\mathrm{P}>0.05)$. HR in the dexmedetomidine group at T2 and T3 was significantly lower than that in the tramadol group, and the differences were statistically significant $(\mathrm{t}=7.306, \mathrm{P}<0.001 ; \mathrm{t}=3.117, \mathrm{P}=0.002)$. The difference of $\mathrm{HR}$ in the dexmedetomidine group was statistically significant $(\mathrm{F}=44.770, \mathrm{P}<0.001)$. HR at $\mathrm{T} 2$ was significantly lower than those at T1 and T3, and HR at T3 was lower than that at T1, and the differences were statistically significant $(\mathrm{P}<0.05)$. There was no significant fluctuation in HR at each time point in the tramadol group, and the differences were not statistically significant $(\mathrm{P}>0.05)$ (Fig. 1).

Levels of MAP in the dexmedetomidine and the tramadol group at different time points. MAP of patients in the dexmedetomidine group at T1, T2 and T3 was 92.38 $\pm 8.45,84.41 \pm 7.32$ and $88.76 \pm 7.72 \mathrm{mmHg}$, respectively, while in the tramadol group was $91.87 \pm 8.13,87.75 \pm 7.69$ and $89.34 \pm 8.07 \mathrm{mmHg}$, respectively. There was no significant difference in MAP between the two groups at T1 and T3 ( $>0.05)$. MAP in the dexmedetomidine group at T2 was significantly lower than that in the tramadol group, and the difference was statistically significant $(\mathrm{t}=3.836$, $\mathrm{P}<0.001)$. There was a statistically significant difference in 
Table I. Basic data of patients in the dexmedetomidine and tramadol groups [n (\%)].

\begin{tabular}{|c|c|c|c|c|}
\hline Factors & Dexmedetomidine group $(n=91)$ & Tramadol group $(\mathrm{n}=85)$ & $\chi^{2}$ value & P-value \\
\hline Sex & & & 0.228 & 0.633 \\
\hline Male & $59(64.84)$ & $58(68.24)$ & & \\
\hline Female & $32(35.16)$ & $27(31.76)$ & & \\
\hline Age (years) & & & 0.389 & 0.533 \\
\hline$\leq 45$ & $25(27.47)$ & $27(31.76)$ & & \\
\hline$>45$ & $66(72.53)$ & $58(68.24)$ & & \\
\hline Weight (kg) & & & 1.344 & 0.246 \\
\hline$\leq 60$ & $36(39.56)$ & $41(48.24)$ & & \\
\hline$>60$ & $55(60.44)$ & $44(51.76)$ & & \\
\hline Alcohol abuse & & & 0.641 & 0.423 \\
\hline Yes & $28(30.77)$ & $31(36.47)$ & & \\
\hline No & $63(69.23)$ & $54(63.53)$ & & \\
\hline No. of tumors & & & 0.401 & 0.527 \\
\hline Single & $64(70.33)$ & $56(65.88)$ & & \\
\hline Multiple & $27(29.67)$ & $29(34.12)$ & & \\
\hline Cirrhosis & & & 0.225 & 0.635 \\
\hline Yes & $69(75.82)$ & $67(78.82)$ & & \\
\hline No & $22(24.18)$ & $18(21.18)$ & & \\
\hline TNM staging & & & 0.684 & 0.408 \\
\hline Stage I & $58(63.74)$ & $49(57.65)$ & & \\
\hline Stage II & $33(36.26)$ & $36(42.35)$ & & \\
\hline
\end{tabular}

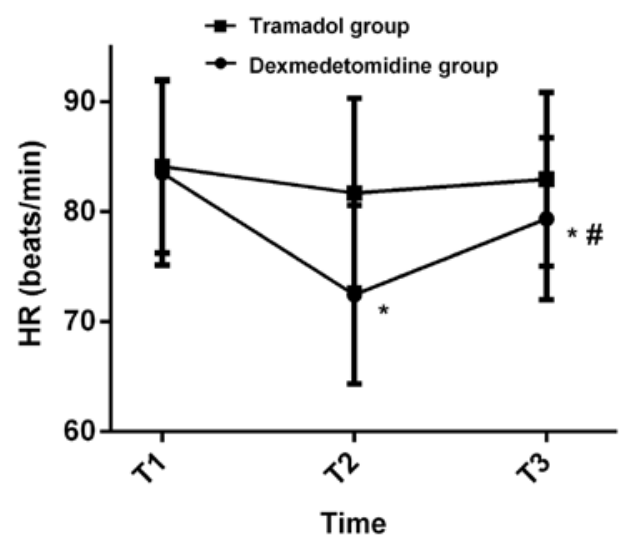

Figure 1. Analysis of HR in the dexmedetomidine and the tramadol group at different time points. There was no significant difference in HR between the two groups at T1 $(\mathrm{P}>0.05)$. HR in the dexmedetomidine group at $\mathrm{T} 2$ and T3 was significantly lower than that in the tramadol group, and the differences were statistically significant $(P<0.05)$. The difference of HRs in the dexmedetomidine group was statistically significant $(\mathrm{P}<0.05)$. HR in the dexmedetomidine group at $\mathrm{T} 2$ was significantly lower than those at $\mathrm{T} 1$ and $\mathrm{T} 3$, and the HR at $\mathrm{T} 3$ was lower than that at $\mathrm{T} 1$, and the differences were statistically significant $(\mathrm{P}<0.05)$. There were no significant fluctuations in $\mathrm{HR}$ at each time point in the tramadol group, and the differences were not statistically significant $(\mathrm{P}>0.05)$. ${ }^{*} \mathrm{P}<0.05$, compared with $\mathrm{T} 1 ;{ }^{*} \mathrm{P}<0.05$, compared with T2. HR, heart rate; T1, before oxygen inhalation; T2, intraoperationally; $\mathrm{T} 3$, at 30 min postoperatively.

MAP in the dexmedetomidine group $(\mathrm{F}=23.550, \mathrm{P}<0.001)$. MAP at T2 was significantly lower than those at $\mathrm{T} 1$ and $\mathrm{T} 3$, and the MAP at T3 was lower than that at T1, and the differences

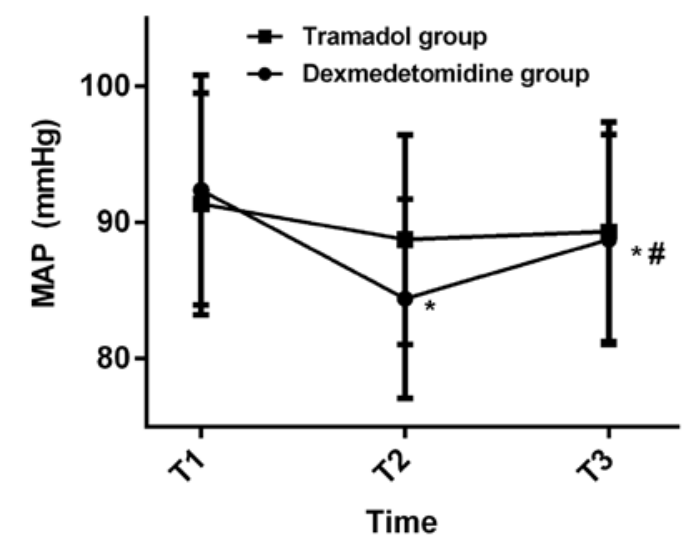

Figure 2. Levels of MAP in the dexmedetomidine and the tramadol group at different time points. There was no significant difference in MAP between the two groups at T1 and T3 ( $>0.05)$. MAP in the dexmedetomidine group at T2 was significantly lower than that in the tramadol group, and the difference was statistically significant $(\mathrm{P}<0.05)$. There was a statistically significant difference in MAP in the dexmedetomidine group $(\mathrm{P}<0.05)$. MAP at T2 was significantly lower than those at T1 and T3, and the MAP at T3 was lower than that at $\mathrm{T} 1$, and the differences were statistically significant $(\mathrm{P}<0.05)$ There was no significant fluctuation in MAP at each time point in the tramadol group, and the differences were not statistically significant $(\mathrm{P}>0.05)$. ${ }^{*} \mathrm{P}<0.05$, compared with $\mathrm{T} 1$; ${ }^{\text {}} \mathrm{P}<0.05$, compared with T2. MAP, mean arterial pressure; T1, before oxygen inhalation; T2, intraoperationally; T3, at $30 \mathrm{~min}$ postoperatively.

were statistically significant $(\mathrm{P}<0.05)$. There was no significant fluctuation in MAP at each time point in the tramadol group, and the differences were not statistically significant $(\mathrm{P}>0.05)$ (Fig. 2). 
Table II. Comparison of recovery time, hospital stay and VAS score between the dexmedetomidine and the tramadol group.

\begin{tabular}{|c|c|c|c|c|}
\hline Factors & Dexmedetomidine group $(n=91)$ & Tramadol group $(n=85)$ & $\mathrm{t}$ value & P-value \\
\hline Recovery time (min) & $7.56 \pm 1.38$ & $7.54 \pm 1.26$ & 0.100 & 0.920 \\
\hline Hospital stay (days) & $14.12 \pm 6.03$ & $13.87 \pm 5.91$ & 0.278 & 0.782 \\
\hline VAS score & $2.87 \pm 0.45$ & $2.62 \pm 0.31$ & 0.853 & 0.395 \\
\hline
\end{tabular}

VAS, visual analogue scale.

Table III. Comparison of adverse reactions between the dexmedetomidine and the tramadol group [n (\%)].

\begin{tabular}{lcccc}
\hline Factors & Dexmedetomidine group $(\mathrm{n}=91)$ & Tramadol group $(\mathrm{n}=85)$ & $\chi^{2}$ value & P-value \\
\hline Bradycardia & $2(2.20)$ & $1(1.18)$ & 0.274 & 0.601 \\
Nausea and vomiting & $3(3.30)$ & $5(5.88)$ & 0.823 & 0.411 \\
Dizziness & $5(5.49)$ & $2(2.35)$ & 1.136 & 0.287 \\
Intraoperative movement & $3(3.30)$ & $1(1.18)$ & 0.690 & 0.406 \\
Shivering & $8(8.79)$ & $1(1.18)$ & 5.252 & 0.022 \\
Total & $21(23.08)$ & $10(11.76)$ & 3.876 & 0.049
\end{tabular}

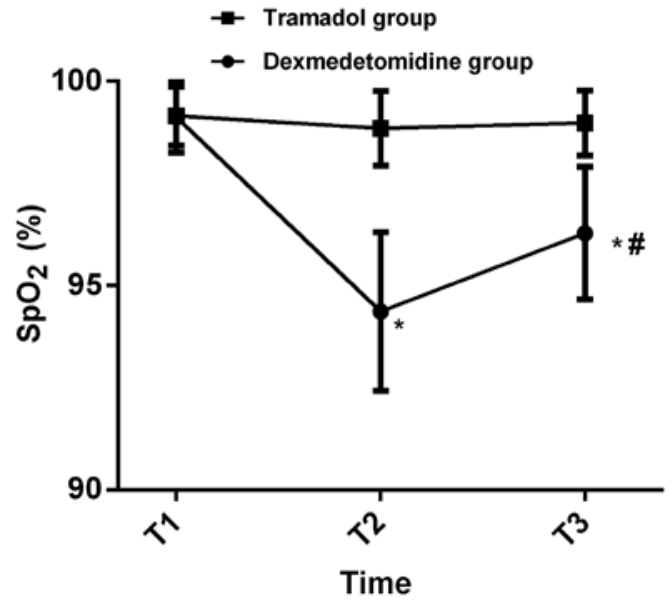

Figure 3. Levels of $\mathrm{SpO}_{2}$ in the dexmedetomidine and the tramadol group at different time points. There was no significant difference in $\mathrm{SpO}_{2}$ between the two groups at $\mathrm{T} 1(\mathrm{P}>0.05) . \mathrm{SpO}_{2}$ in the dexmedetomidine group at $\mathrm{T} 2$ and T3 was significantly lower than that in the tramadol group, and the differences were statistically significant $(\mathrm{P}<0.05)$. The difference of $\mathrm{SpO}_{2}$ in the dexmedetomidine group was statistically significant $(\mathrm{P}<0.05) . \mathrm{SpO}_{2}$ at T2 was significantly lower than those at $\mathrm{T} 1$ and $\mathrm{T} 3$, and the $\mathrm{SpO}_{2}$ at T3 was lower than that at $\mathrm{T} 1$, and the differences were statistically significant $(\mathrm{P}<0.05)$. There was no significant fluctuation in $\mathrm{SpO}_{2}$ at each time point in the tramadol group, and the differences were not statistically significant ( $\mathrm{P}>0.05)$. ${ }^{*} \mathrm{P}<0.05$, compared with $\mathrm{T} 1 ;{ }^{\sharp} \mathrm{P}<0.05$, compared with $\mathrm{T} 2 . \mathrm{SpO}_{2}$, oxygen saturation; T1, before oxygen inhalation; T2, intraoperationally; T3, at $30 \mathrm{~min}$ postoperatively.

Levels of $\mathrm{SpO}_{2}$ in the dexmedetomidine and the tramadol group at different time points. $\mathrm{SpO}_{2}$ of patients in the dexmedetomidine group at T1, T2 and T3 was 99.12 \pm 0.86 , $94.36 \pm 1.94$ and $96.28 \pm 1.62 \%$, respectively. $\mathrm{SpO}_{2}$ of patients in the tramadol group at different time points was $99.15 \pm 0.72$, $98.85 \pm 0.91$ and $98.98 \pm 0.79 \%$, respectively. There was no significant difference in $\mathrm{SpO}_{2}$ between the two groups at $\mathrm{T} 1$
( $\mathrm{P}>0.05) . \mathrm{SpO}_{2}$ in the dexmedetomidine group at $\mathrm{T} 2$ and $\mathrm{T} 3$ was significantly lower than that in the tramadol group, and the differences were statistically significant $(\mathrm{t}=19.430, \mathrm{P}<0.001$; $\mathrm{t}=13.900, \mathrm{P}=0.002)$. The difference of $\mathrm{SpO}_{2}$ in the dexmedetomidine group was statistically significant $(\mathrm{F}=219.700$, $\mathrm{P}<0.001) . \mathrm{SpO}_{2}$ at $\mathrm{T} 2$ was significantly lower than those at $\mathrm{T} 1$ and $\mathrm{T} 3$, and $\mathrm{SpO}_{2}$ at $\mathrm{T} 3$ was lower than that at T1, and the differences were statistically significant $(\mathrm{P}<0.05)$. There was no significant fluctuation in $\mathrm{SpO}_{2}$ at each time point in the tramadol group, and the differences were not statistically significant ( $\mathrm{P}>0.05)$ (Fig. 3).

Comparison of recovery time, hospital stay and VAS score between the dexmedetomidine and the tramadol group. There was no significant difference in recovery time, hospital stay and VAS score between the two groups ( $\mathrm{P}>0.05)$ (Table II).

Comparison of perioperative complications between the dexmedetomidine and the tramadol group. The number of patients with shivering in dexmedetomidine group was significantly higher than that in tramadol group, and the difference was statistically significant $(\mathrm{P}<0.05)$. There was no significant difference in the number of patients with bradycardia, nausea and vomiting, dizziness, or intraoperative movement between the dexmedetomidine and the tramadol group (P>0.05) (Table III).

\section{Discussion}

There is still global disparity in the incidence of HCC, and East and Southeast Asia remain with the highest incidence. However, although, in recent years in most of these high-risk countries the incidence has been declining, the morbidity in several low-risk countries in Europe, America and Oceania 
is steadily increasing, due to the fact that obesity, type 2 diabetes and non-alcoholic fatty liver diseases are replacing viral and alcoholic liver diseases to become major pathogenic factors (14). The median survival time of late $\mathrm{HCC}$ has been reported to be $<1$ year and the 5-year survival rate $<10 \%$ (15). Therefore, the early diagnosis and treatment are very important for the improvement of the prognosis of patients. In recent years, PMCT has become an important method and has been widely used in the comprehensive treatment of HCC because of its minimal invasion, high safety and efficacy (16). Ultrasound-guided PMCT has a faster overall heating rate and less trauma, which can effectively alleviate or eliminate the clinical symptoms after treatment of HCC patients, and therefore is widely used in clinical practice $(17,18)$. In the process of PMCT, the choice of anesthetic is the key to stabilize vital signs and reduce postoperative adverse reactions.

In this study, the dosage of anesthetic drugs was set according to patients' weight and age, and the changes of intraoperative $\mathrm{HR}, \mathrm{MAP}$ and $\mathrm{SpO}_{2}$ indexes in patients were observed. It was found that the intraoperative HR, MAP, and $\mathrm{SpO}_{2}$ in the dexmedetomidine group were significantly lower than those in the tramadol group, and their fluctuation differences were significantly higher than those in the tramadol group. Dexmedetomidine is a highly selectivity new $\alpha_{2}$ adrenergic receptor agonist, the activation of which in the brain and spinal cord can inhibit neuronal excitation, thereby leading to hypotension, bradycardia and other hemodynamic changes $(19,20)$. It can produce an analgesic effect through the synaptic and posterior synaptic membrane $\alpha_{2}$ receptors in the spinal cord interneurons to inhibit central sympathetic nerves, enhance vagal activity, and weaken the sympathetic tone, thus having a better analgesic effect (21).

In the study of hemodynamic response of dexmedetomidine in patients undergoing laparoscopic cholecystectomy by Bhagat et al (22), it was found that dexmedetomidine was associated with hypotension and bradycardia. There were 5 cases of bradycardia in dexmedetomidine group, all of which needed to be injected with atropine. The binding of tramadol to opioid receptor could inhibit hypothalamic thermoregulatory center and re-uptake of spinal cord 5-hydroxytryptamine and methylepinephrine, increase the concentration of methylepinephrine and 5-hydroxytryptamine synaptosomes of the spinal cord, and regulate the body temperature, thus effectively prevent shivering reaction. However, it showed more gastrointestinal tract reaction and higher incidence of nausea $(23,24)$. These results support our study that the number of patients with shivering in the dexmedetomidine group was significantly higher than that in the tramadol group, while the incidence of nausea and vomiting in the tramadol group was higher than that in the dexmedetomidine group. According to the study by Bedirli et al (25), both tramadol and dexmedetomidine are effective in controlling pain and restlessness. However, compared with tramadol, dexmedetomidine might cause intraoperative hypotension, bradycardia and long-term sedation. According to Sahi et al (26), tramadol plays the most important role in preventing shivering. Although it has a higher incidence of nausea and vomiting than clonidine and dexmedetomidine, these symptoms can be alleviated without intervention in most patients. Moreover, tramadol was reported to have the least side-effects.
In the present study, due to the limited medical resources in The Affiliated Hospital of Qingdao University and the small base of the selected research subjects, there may be some contingency in the results, and sex or age differences in postoperative responses to anaesthesia cannot be excluded.

In conclusion, both tramadol and dexmedetomidine, respectively, combined with propofol in PMCT for HCC surgery can achieve satisfactory anesthetic effects. However, tramadol combined with propofol is more effective in stabilizing the vital signs with less side-effects, and more suitable for PMCT in patients with HCC than dexmetomidine combined with propofol.

\section{Acknowledgements}

Not applicable.

\section{Funding}

No funding was received.

\section{Availability of data and materials}

The datasets used and/or analyzed during the present study are available from the corresponding author on reasonable request.

\section{Authors' contributions}

CC and XY were responsible for the treatment of patients. JS and $\mathrm{XZ}$ collected and analyzed the general data of patients. SL and NZ analyzed the changes in HR, MAP, and $\mathrm{SpO}_{2} . \mathrm{CC}$ and JW performed the statistical analysis. CC drafted the manuscript. All authors read and approved the final manuscript.

\section{Ethics approval and consent to participate}

The study was approved by the Ethics Committee of The Affiliated Hospital of Qingdao University (Qingdao, China). Patients who participated in this research had complete clinical data. Signed informed consents were obtained from the patients and their guardians.

\section{Patient consent for publication}

Not applicable.

\section{Competing interests}

The authors declare that they have no competing interests.

\section{References}

1. Marquardt JU, Andersen JB and Thorgeirsson SS: Functional and genetic deconstruction of the cellular origin in liver cancer. Nat Rev Cancer 15: 653-667, 2015.

2. Torre LA, Bray F, Siegel RL, Ferlay J, Lortet-Tieulent J and Jemal A: Global cancer statistics, 2012. CA Cancer J Clin 65: 87-108, 2015.

3. Tsai WC, Kung PT, Wang YH, Kuo WY and Li YH: Influence of the time interval from diagnosis to treatment on survival for early-stage liver cancer. PLoS One 13: e0199532, 2018.

4. Li L and Wang H: Heterogeneity of liver cancer and personalized therapy. Cancer Lett 379: 191-197, 2016. 
5. Kodack DP, Farago AF, Dastur A, Held MA, Dardaei L, Friboulet L, von Flotow F, Damon LJ, Lee D, Parks M, et al: Primary patient-derived cancer cells and their potential for personalized cancer patient care. Cell Rep 21: 3298-3309, 2017.

6. Facciorusso A, Di Maso M and Muscatiello N: Microwave ablation versus radiofrequency ablation for the treatment of hepatocellular carcinoma: A systematic review and meta-analysis. Int J Hyperthermia 32: 339-344, 2016

7. Amabile C, Ahmed M, Solbiati L, Meloni MF, Solbiati M, Cassarino $\mathrm{S}$, Tosoratti $\mathrm{N}$, Nissenbaum $\mathrm{Y}$, Ierace $\mathrm{T}$ and Goldberg SN: Microwave ablation of primary and secondary liver tumours: Ex vivo, in vivo, and clinical characterisation. Int J Hyperthermia 33: 34-42, 2017.

8. Ryan MJ, Willatt J, Majdalany BS, Kielar AZ, Chong S, Ruma JA and Pandya A: Ablation techniques for primary and metastatic liver tumors. World J Hepatol 8: 191-199, 2016.

9. Dou JP, Liang P and Yu J: Microwave ablation for liver tumors Abdom Radiol (NY) 41: 650-658, 2016.

10. Freeman J and Buggy DJ: Modelling the effects of perioperative interventions on cancer outcome: Lessons from dexmedetomidine. Br J Anaesth 120: 15-17, 2018.

11. Miotto K, Cho AK, Khalil MA, Blanco K, Sasaki JD and Rawson R: Trends in tramadol: Pharmacology, metabolism, and misuse. Anesth Analg 124: 44-51, 2017.

12. World Health Organization: Expert Committee on Drug Dependence (ECDD) 34th Report. Assessment of tramadol, 2006.

13. Jin $Q$, Chen $X$ and Zheng S: The security rating on local ablation and interventional therapy for hepatocellular carcinoma (HCC) and the comparison among multiple anesthesia methods. Anal Cell Pathol (Amst) 2019: 2965173, 2019.

14. Petrick JL, Braunlin M, Laversanne M, Valery PC, Bray F and McGlynn KA: International trends in liver cancer incidence, overall and by histologic subtype, 1978-2007. Int J Cancer 139: $1534-1545,2016$

15. Trevisani F, Cantarini MC, Wands JR and Bernardi M: Recent advances in the natural history of hepatocellular carcinoma. Carcinogenesis 29: 1299-1305, 2008.

16. Xu LF, Chen YT, Ren Q, Ma HQ, Zhang J, Chen B, Lu PX and Tan ZY: Clinical application of transarterial chemoembolization combining with air-cooled percutaneous microwave coagulation therapy on patients with primary hepatic carcinoma. Chin J Clin Oncol 34: 553-557, 2007 (In Chinese).

17. Potretzke TA, Ziemlewicz TJ, Hinshaw JL, Lubner MG, Wells SA, Brace CL, Agarwal P and Lee FT Jr: Microwave versus radiofrequency ablation treatment for hepatocellular carcinoma: A comparison of efficacy at a single center. J Vasc Interv Radiol 27: 631-638, 2016
18. Jiang X, Gao F, Ma Y, Feng S, Liu X and Zhou H: Percutaneous microwave ablation in the spleen for treatment of hypersplenism in cirrhosis patients. Dig Dis Sci 61: 287-292, 2016.

19. Panchgar V, Shetti AN, Sunitha HB, Dhulkhed VK and Nadkarni AV: The effectiveness of intravenous dexmedetomidine on perioperative hemodynamics, analgesic requirement, and side effects profile in patients undergoing laparoscopic surgery under general anesthesia. Anesth Essays Res 11: 72-77, 2017.

20. Hoy SM and Keating GM: Dexmedetomidine: A review of its use for sedation in mechanically ventilated patients in an intensive care setting and for procedural sedation. Drugs 71: 1481-1501, 2011.

21. Weerink MAS, Struys MMRF, Hannivoort LN, Barends CRM, Absalom AR and Colin P: Clinical pharmacokinetics and pharmacodynamics of dexmedetomidine. Clin Pharmacokinet 56: 893-913, 2017.

22. Bhagat N, Yunus M, Karim HM, Hajong R, Bhattacharyya P and Singh M: Dexmedetomidine in attenuation of haemodynamic response and dose sparing effect on opioid and anaesthetic agents in patients undergoing laparoscopic cholecystectomy - A randomized study. J Clin Diagn Res 10: UC01-UC05, 2016.

23. Falls BA: Opioids and serotonin syndrome: An example with tramadol. In: Neuropathology of Drug Addictions and Substance Misuse - General Processes and Mechanisms, Prescription Medications, Caffeine and Areca, Polydrug Misuse, Emerging Addictions and Non-Drug Addictions. Preedy VR (ed). Vol 3. Elsevier Inc., Academic Press, London, pp443-448, 2016.

24. Faria J, Barbosa J, Queirós O, Moreira R, Carvalho F and Dinis-Oliveira RJ: Comparative study of the neurotoxicological effects of tramadol and tapentadol in SH-SY5Y cells. Toxicology 359-360: 1-10, 2016.

25. Bedirli N, Akçabay M and Emik U: Tramadol vs dexmedetomidine for emergence agitation control in pediatric patients undergoing adenotonsillectomy with sevoflurane anesthesia: Prospective randomized controlled clinical study. BMC Anesthesiol 17: 41, 2017.

26. Sahi S, Singh MR and Katyal S: Comparative efficacy of intravenous dexmedetomidine, clonidine, and tramadol in postanesthesia shivering. J Anaesthesiol Clin Pharmacol 32: 240-244, 2016.

This work is licensed under a Creative Commons Attribution-NonCommercial-NoDerivatives 4.0 International (CC BY-NC-ND 4.0) License. 